\title{
Die Historische Sondersammlung « Soziale Arbeit und Pflege »
}

Un centre de documentation et une bibliothèque spécialisés dans l'histoire du soin et du travail social à Francfort

\section{Emmanuel Delille}

\section{(2) OpenEdition \\ Journals}

Édition électronique

URL : http://journals.openedition.org/ifha/167

DOI : $10.4000 /$ ifha. 167

ISSN : 2198-8943

Éditeur

IFRA - Institut franco-allemand (sciences historiques et sociales)

Édition imprimée

Date de publication : 6 février 2011

Pagination : $95-100$

ISSN : 2190-0078

\section{Référence électronique}

Emmanuel Delille, «Die Historische Sondersammlung « Soziale Arbeit und Pflege » », Revue de l'IFHA [En ligne], 3 | 2011, mis en ligne le 10 février 2012, consulté le 01 mai 2019. URL : http:// journals.openedition.org/ifha/167; DOI : 10.4000/ifha.167

Ce document a été généré automatiquement le 1 mai 2019.

(CIFHA 


\section{Die Historische Sondersammlung "Soziale Arbeit und Pflege "}

Un centre de documentation et une bibliothèque spécialisés dans l'histoire du soin et du travail social à Francfort

\section{Emmanuel Delille}

\section{NOTE DE L'ÉDITEUR}

Emmanuel Delille est, depuis 2007, chercheur associé au centre Marc-Bloch (Berlin). Il a bénéficié d'une bourse de courte durée de l'IFHA en juillet-août 2011.

\section{NOTE DE L'AUTEUR}

Adresse géographique :

Historische Sondersammlung « Soziale Arbeit und Pflege »

Kleiststraße 31 (bâtiment 3, 4e étage, salles 414-415)

D-60 318 Frankfurt am Main

Interlocutrice :

Inke Worgitzki (coordinatrice des collections)

Tél. : 0049 (0) 69/153 32847 ou 622

Fax : 0049 (0) 69/153 32465

Courriel : histo@bibl.fh-frankfurt.de

Internet, avec un lien vers le catalogue en ligne des collections spécialisées :

http://www.fh-frankfurt.de/de/service_fuer_studierende/bibliothek/

historische_sondersammlung.html

La Fachhochschule de Francfort-sur-le-Main abrite dans sa bibliothèque un centre de documentation unique en Allemagne: un lieu spécialisé dans l'histoire du soin et du travail social. Bien sûr, il existe dans tous les länder allemands des bibliothèques 
rattachées aux écoles de formation des infirmiers et des travailleurs sociaux, à côté des bibliothèques d'histoire de la médecine, susceptibles d'offrir une documentation importante et facilement accessible aux historiens. Mais la Historische Sondersammlung "Soziale Arbeit und Pflege» a ceci de particulier qu'elle a été créée comme un centre de documentation historique, et que ses collections regroupent et centralisent plusieurs fonds privés ou d'associations professionnelles allemandes: les archives et fonds de l'association allemande pour les soins publics et privés (Deutscher Verein für öffentliche und private Fürsorge); la bibliothèque de l'ancien séminaire de l'université de Francfort sur l'histoire du soin ; le fonds ancien du centre de documentation constitué par la donation de Hilde Steppe (connu sous le nom d'archives Hilde-Steppe, Hilde-Steppe-Archiv); les livres historiques et les revues des deux disciplines «santé » et «travail social», conservés à la bibliothèque de la Fachhochschule de Francfort.

\section{Description du fonds}

Les collections regroupent près de 15000 monographies, ouvrages publiés, travaux d'étudiants et littérature grise, concernant le travail social ; près de 4000 monographies, ouvrages publiés, travaux d'étudiants et littérature grise sur l'histoire du soin infirmier dans les pays de langue allemande à l'époque contemporaine (Allemagne[s], Autriche et Suisse) ; environ 200 revues et journaux spécialisés ; des fonds privés issus de donations posthumes; une petite quantité d'objets qui ont acquis un statut muséal (vêtements et insignes d'infirmières, etc.) ; enfin, quelques ressources iconographiques sur l'histoire du soin infirmier (photographies, cartes postales, etc.).

Cette description succincte des collections indique déjà deux caractéristiques historiographiques et archivistiques fortes du centre de documentation: un fonds construit pour enseigner et documenter une mémoire professionnelle contemporaine (XIXe et XXe siècles) et créé sur une initiative personnelle, celle de H. Steppe. Ses archives (le Nachlass Hilde Steppe) forment le noyau dur des collections. Un inventaire établi en 2000-2001, sous la direction d'Eva Maria Ulmer, décrit et recense les archives conservées selon quatre types de classements - numérique, thématique, chronologique et alphabétique. Cet inventaire est communiqué aux chercheurs sur demande.

\section{Une histoire professionnelle}

H. Steppe étant pour l'essentiel à l'origine du centre de documentation historique spécialisé, il convient de lui consacrer une place particulière. Née en 1947 en RFA et décédée en 1999, elle fit sa formation d'infirmière entre 1965 et 1968. Elle occupa divers postes en Allemagne de l'Ouest, notamment à l'Institut d'anesthésiologie de la clinique universitaire de Tübingen. En 1978, elle devint enseignante à Francfort au Centre de formation pour les métiers de santé (Fortbildungszentrum für Berufe im Gesundheitswesen) et, rapidement, accéda à un poste de direction. Elle commence alors à construire, au début des années 1980, l'esquisse de ce qui va devenir les archives sur l'histoire du soin. Elle recueille et collectionne systématiquement des sources susceptibles de documenter l'histoire de professions en règle générale féminines et peu valorisées dans l'histoire des savoirs, celles des infirmières et sœurs infirmières: livres anciens ou contemporains, documents professionnels ou mémoriels, au croisement de l'histoire du soin et de l'histoire des femmes, en particulier pendant la période national-socialiste ${ }^{1}$. Elle 
développe et intègre l'histoire orale à son projet historiographique, complétant les recherches déjà existantes sur ces acteurs souvent considérés comme de simples intermédiaires, les infirmières, alors que l'on ne peut pourtant classer leur rôle complexe ${ }^{2}$ dans la seule catégorie des témoins, ni dans celle des exécuteurs ou victimes - pour reprendre le découpage conceptuel bien connu de Raul Hilberg. Ce travail fit de H. Steppe une figure centrale de la recherche sur l'histoire du soin en Allemagne. La documentation accumulée prit la forme matérielle d'un centre de documentation à partir de 1995.

En complément de son travail archivistique, H. Steppe eut le souci de la transmission, poursuivit sa formation universitaire, publia des résultats de recherche et encouragea des travaux collectifs grâce à la création d'un groupe de travail sur l'histoire du soin ( Arbeitsgemeinschaft zur Geschichte der Pflege) ${ }^{3}$. Diplômée en pédagogie en 1994, docteure en 1997 avec une thèse portant sur l'histoire des infirmières et institutions de soins juives ${ }^{4}$, elle fut immédiatement appelée au poste de professeure de la Fachhochschule de Francfort. $\mathrm{H}$. Steppe joua également un rôle politique auprès du ministère de la Santé du land de Hesse de 1992 à 1998 (lors de la réforme et codification de la formation d'infirmière) et son œuvre fut récompensée par des prix.

\section{L'histoire du soin dans la communauté juive de Francfort}

À noter aussi qu'un projet de recherche lié au centre de documentation et à la Fachhochschule est consacré à l'histoire du soin dans la communauté juive de Francfort ${ }^{5}$, la ville ayant longtemps abrité une des plus grandes communautés juives des pays de langue allemande, depuis le Moyen Âge jusqu'aux déportations de 1941-19426. Cette communauté s'était dotée de ses propres institutions et services de santé, attestés depuis le XVe siècle. Le projet se décline sur deux axes, l'un prosopographique et l'autre sur l'histoire des institutions. Il est soutenu et animé par des enseignants-chercheurs - notamment par E.M. Ulmer -, des fondations et des associations professionnelles. Le site internet est directement accessible par des liens hypertextes depuis le site de la Fachhochschule de Francfort et, surtout, donne accès aux notices biographiques rédigées à ce jour, pour la période 1870-1945. Des pages interactives présentent aussi une carte des institutions juives de Francfort, une chronologie et une sélection iconographique.

\section{Culture mémorielle, biais archivistiques, intérêt historiographique}

La première critique qu'un historien peut faire du point de vue de la construction archivistique, lorsqu'il est confronté au type de fonds élaboré par H. Steppe et ses collègues, porte avant tout sur les biais induits par la culture mémorielle et la recherche d'identité d'une profession, en quête de son histoire. Toutefois, les outils développés par le centre de documentation permettent aussi d'y remédier: d'une part, les collections issues du Nachlass de H. Steppe sont décrites de manière exhaustive dans un catalogue papier d'une centaine de pages, à partir duquel les chercheurs peuvent vérifier la provenance, l'ancienneté et la nature des documents consultables; d'autre part, le fonds est regroupé avec les autres ouvrages de la bibliothèque de la Fachhochschule de Francfort dans un catalogue informatique unique, ce qui permet à la fois d'élargir toute enquête à 
des ouvrages et documents récents qui ne font pas partie des collections spécialisées, et en même temps de vérifier aussitôt par un système de cote précis quels sont les documents d'archive. Soulignons également que l'inventaire du Nachlass fait apparaître des thèmes transversaux communs à l'histoire sociale, politique et culturelle, comme l'enfance, la mort, la guerre, le genre, l'Église, le judaïsme, le national-socialisme, etc., thèmes qui dépassent largement le cadre de l'enseignement de la théorie du soin (care) et des batailles professionnelles du personnel infirmier. L'inventaire fait aussi ressortir un nombre important de correspondances, d'entretiens conservés sur support audio ou vidéo, ainsi que des retranscriptions d'entretiens, des coupures de presse ou des articles scientifiques classés de manière thématique.

\section{La sécularisation des savoir-faire, entre science et fiction}

Point fort à relever, les collections comprennent un nombre estimable de biographies et de mémoires, voire de fictions et de romans, écrits par (ou à destination) des infirmières, et surtout par (ou à destination) des sœurs et des acteurs importants des ordres religieux qui ont fait l'histoire du soin dans les pays de langue allemande. Autre particularité intéressante - soit conséquence du choix des sources, soit résultat d'une culture distincte de la mémoire -, les ordres chrétiens réformés (les diaconesses, pour être précis) semblent à ce titre avoir laissé plus de témoignages écrits que les ordres catholiques. Des manuels d'éthique ou d'enseignement pour la formation des sœurs aux soins infirmiers, ayant parfois bénéficié d'un nombre honorable de rééditions au fil du temps, témoignent du processus de sécularisation des savoirs et des pratiques sur la longue durée. N’oublions pas les témoignages sur la guerre (en particulier la Grande Guerre), sur les missions catholiques, protestantes et laïques, sur les institutions coloniales et enfin sur les organismes internationaux (Croix-Rouge), qui traversent ce type de récits de vie et d'itinéraires professionnels dans le siècle. Le système d'indexation informatique des ouvrages par mots-clés favorise la surreprésentation des ouvrages publiés ces dernières décennies, au détriment des livres plus anciens, mais ce problème n'est pas spécifique au champ du soin et embarrasse tous les historiens! Ces remarques critiques veulent au contraire souligner l'intérêt, pour les chercheurs français, de consulter la base de données de la Historische Sondersammlung "Soziale Arbeit und Pflege», car ils trouveront rarement, pour les pays francophones, un fonds riche et un centre de documentation doté d'équipements modernes aussi accessibles que peuvent l'être ceux de Francfort.

\section{NOTES}

1. Hilde Steppe (dir.), Krankenpflege im Nationalsozialismus, 10e éd., Frankfurt a.M. : Mabuse, 2011. 
2. H. Steppe, E.M. Ulmer (dir.), « Ich war von jeher mit Leib und Seele gerne Pflegerin. » Über die Beteiligung von Krankenschwestern an den "Euthanasie"-Aktionen in MeseritzObrawalde, 3e éd., Frankfurt a.M. : Mabuse, 2010.

3. Groupe de travail à l'origine de la publication collective Geschichte der Krankenpflege. Versuch einer kritischen Aufarbeitung, Berlin, 1984.

4. H. Steppe, « Den Kranken zum Troste und dem Judentum zur Ehre. » Zur Geschichte der jüdischen Krankenpflege in Deutschland, Frankfurt a.M. : Mabuse, 1997.

5. Projet « Jüdische Pflegegeschichte », http://www.juedische-pflegegeschichte.de.

6. Histoire notamment retracée au musée Juif de Francfort, qui n'est pas seulement un lieu d'exposition, mais aussi un centre de documentation, acteur et soutien de la recherche scientifique. 